\title{
Fotógrafo de estrellas - Star Photographer
}

$\begin{aligned} \text { Authors: } & \text { Valentino Beorda } \\ \text { Submitted: } & \text { 18. August } 2021 \\ \text { Published: } & 23 . \text { August } 2021 \\ \text { Volume: } & 8 \\ \text { Issue: } & 4 \\ \text { Affiliation: } & \text { El Gato y la Caja, Buenos Aires, Argentina } \\ \text { Languages: } & \text { Spanish, Castilian } \\ \text { Keywords: } & \text { Hubble, Space, Telescope, Universe, Photography, Stars } \\ \text { Categories: } & \text { News and Views } \\ \text { DOI: } & 10.17160 / \text { josha.8.4.778 }\end{aligned}$

Abstract:

More than 30 years have passed since the Hubble Space Telescope was put into orbit at $7 \mathrm{~km}$ per second and $500 \mathrm{~km}$ far from the Earth surface. After a few (not so minor) adjustments, the Hubble Telescope gathered data that has been published in more than 15,000 scientific articles. On the contrary of what was hypothesized during the late 90s, the Hubble Telescope was critical for determining that the universe is expanding, and that the expansion velocity is actually increasing. This also supported the idea of the existence of dark energy and dark matter, something that we still do not understand at all, but occupies 68 and $27 \%$ of the Universe, respectively. It is expected that the Hubble Telescope will be operational until 2030, and then replaced by the James Webb Space Telescope. The Hubble Telescope gave us the most striking images we have from our Universe so far. This is it's story. 


\title{
Fotógrafo de estrellas - Star Photographer
}

\author{
Valentino Beorda \\ El Gato y La Caja \\ Buenos Aires, Argentina
}

Ilustradora: Margarita Cubino

Fecha de publicación: 20/4/20

Link a la nota original: https://elgatoylacaja.com/fotografo-de-estrellas/

\section{Abstract}

More than 30 years have passed since the Hubble Space Telescope was put into orbit at $7 \mathrm{~km}$ per second and $500 \mathrm{~km}$ far from the Earth surface. After a few (not so minor) adjustments, the Hubble Telescope gathered data that has been published in more than 15,000 scientific articles. On the contrary of what was hypothesized during the late 90s, the Hubble Telescope was critical for determining that the universe is expanding, and that the expansion velocity is actually increasing. This also supported the idea of the existence of dark energy and dark matter, something that we still do not understand at all, but occupies 68 and $27 \%$ of the Universe, respectively. It is expected that the Hubble Telescope will be operational until 2030, and then replaced by the James Webb Space Telescope. The Hubble Telescope gave us the most striking images we have from our Universe so far. This is it's story. 
Corría el año 1609 cuando Galileo Galilei escuchó acerca de un patentamiento enviado al gobierno de Holanda por el inventor Hans Lippershey, que consistía en un instrumento "para ver cosas lejanas como si estuvieran cerca". No sabemos cómo logró hacerse del diseño original, pero lo cierto es que le aplicó ciertas mejoras y construyó entonces su propio telescopio. Así, empezó a observar el cosmos. Descubrió las cuatro lunas más grandes de Júpiter, hoy conocidas como 'satélites galileanos', y con esto demostró que, contrario a lo que se creía en esa época, no todo giraba alrededor de la Tierra.

Así como Galileo, muchos otros astrónomos han contribuido al entendimiento de nuestro Universo a partir de la observación astronómica. Con el paso de los años, el avance de la ciencia y la tecnología han permitido construir instrumentos más precisos y sofisticados que nos ayudan a conocer cada día un poco más del plano general más general que tenemos. Una de estas herramientas es el Telescopio Espacial Hubble, que el 24 de abril cumplió 30 años en órbita, y el cual ha ayudado a calcular la edad del Universo y su radio de expansión, ratificar que casi todas las galaxias tienen un agujero negro en su centro, crear un mapa 3D de la distribución de la materia oscura en el Universo, y muchas cosas más.

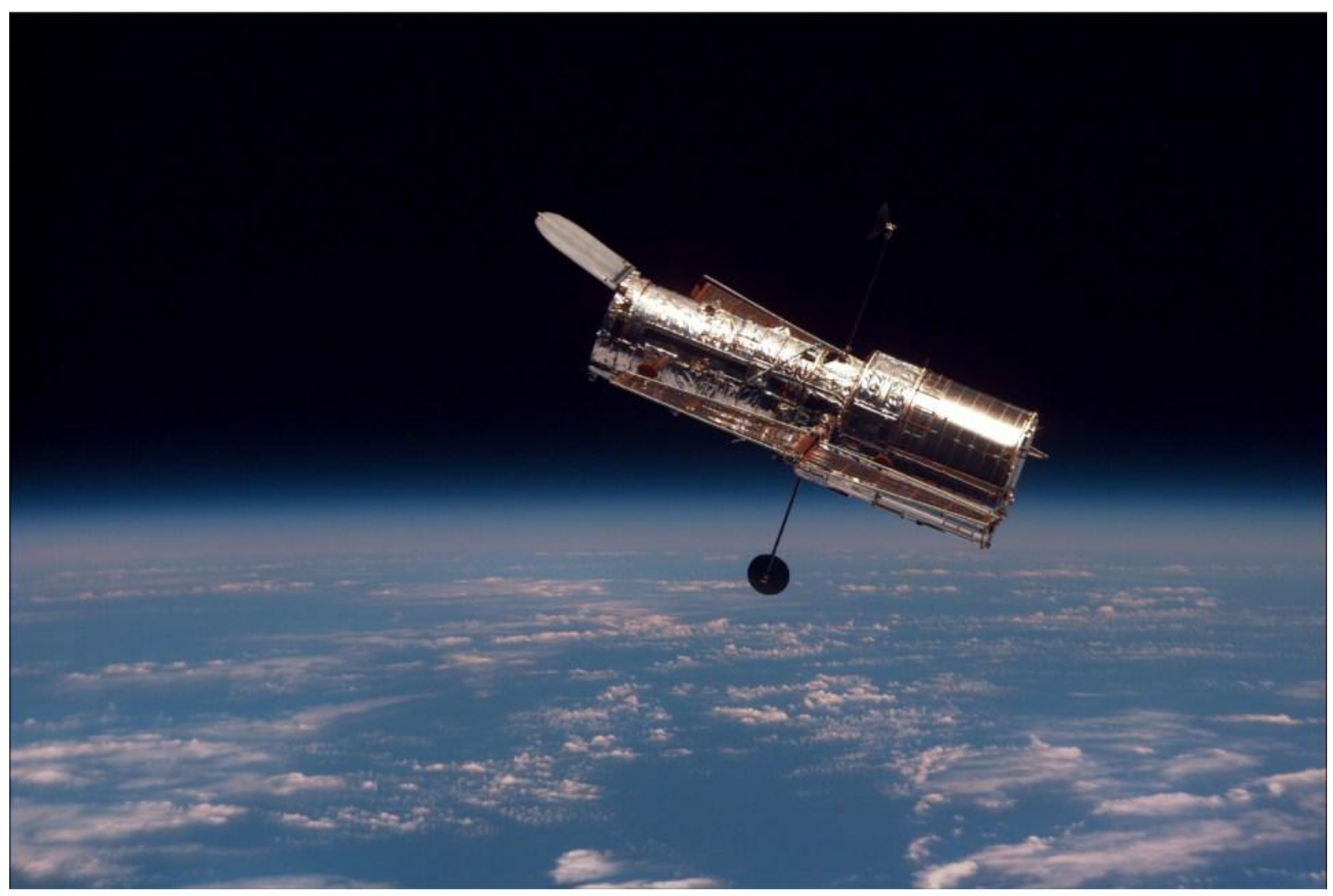




\section{¡Un brindis por el cumpleañero! Crédito: NASA}

Hermoso, ¿no? Parece un rollo de papel aluminio con accesorios. Pero ¿qué hace ahí arriba, solo, flotando en el umbral del vacío? ¿Qué motiva el envío de un telescopio al espacio, siendo que tranquilamente lo podríamos mantener sano y salvo dentro de un observatorio aquí en la Tierra? El principal problema de observar el cosmos desde la superficie terrestre es nuestra atmósfera, que si bien tiene algunas ventajas como permitirnos estar vivos, en lo que a observar el Universo respecta no es tan amigable, ya que distorsiona de forma importante las imágenes que se obtienen desde un telescopio, culpa de un efecto denominado 'aberración óptica'. Y como si esto fuera poco, también se encarga de absorber una gran porción del espectro electromagnético, lo cual en pocas palabras significa que no deja 'pasar' muchas ondas infrarrojas, ultravioletas, rayos $\mathrm{X}$, etc., muy importantes para el descubrimiento de nuevos objetos astronómicos. 


\section{Penetra la atmósfera}

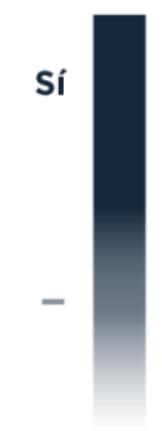

No

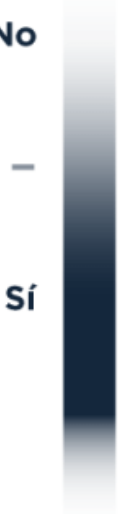

No

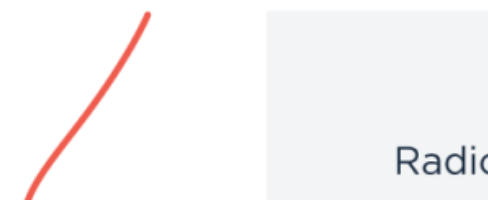

$10^{3}$

Long. de onda (m)

\section{Escala aproximada}

Tipo

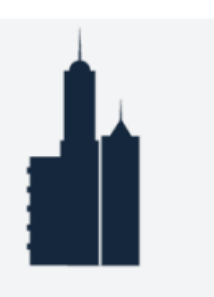

Microondas

$10^{-2}$

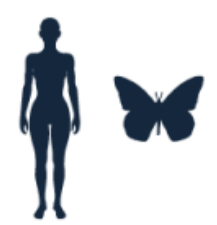

Infrarrojo

$10^{-5}$

Visible

$0,5 \times 10^{-6}$

Ultravioleta

$10^{-8}$

Rayos X

$10^{-10}$

$\odot$

Rayos

gamma

$10^{-12}$

Todas las ondas que componen el espectro electromagnético.

En 1946 el astrónomo Lyman Spitzer publicó un artículo en el que hablaba acerca de las ventajas de enviar un telescopio al espacio. La idea no era original, ya 20 años antes otro artículo soñaba con la misma posibilidad. Pero para Spitzer la obsesión por los telescopios 
espaciales fue muy grande y dedicó gran parte de su carrera a impulsar su desarrollo. En 1962 lideró un programa para diseñar un observatorio que orbitara la Tierra y estudiara la luz ultravioleta del espacio, el cual se convirtió en el exitoso programa Orbiting Astronomical Observatory (OAO) de la NASA, que consistió en cuatro observatorios espaciales. Dos se lograron lanzar entre 1966 y 1972 y proporcionaron las primeras observaciones de alta calidad de muchos objetos astronómicos en luz ultravioleta.

Pero el diseño de un telescopio espacial con las cualidades que tendría el Hubble empezó en 1965, recomendado por la Academia Nacional de Ciencias, en un comité también liderado por Lyman Spitzer, que tenía el propósito de definir los objetivos científicos para dicho telescopio. $Y$ vaya que los definieron: el Hubble fue construido con el objetivo de descifrar, entre otras cosas, la edad del Universo y su velocidad de expansión. Tranqui. El nombre se lo pusieron entonces en conmemoración a Edwin Hubble, quien en 1924 presentó las primeras pruebas acerca de la teoría de Georges Lemaître, la cual afirma que el Universo está en estado de expansión. 


\section{JOSHA}

Journal of Science, Humanities and Arts

August 2021

Volume 8, Issue 4

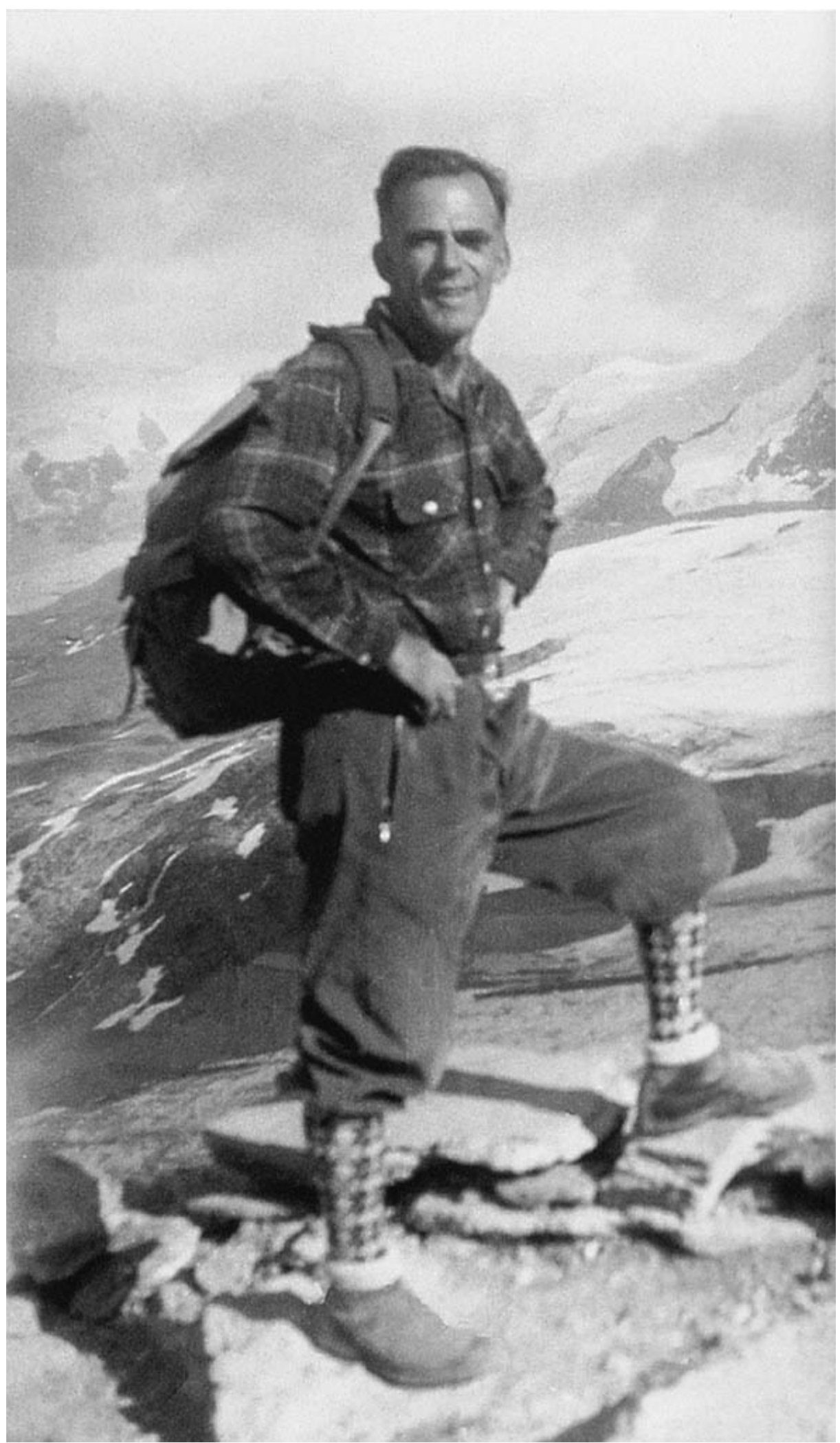




\section{Spitzer, listo para la aventura}

Como en los '60, la NASA venía haciendo varias investigaciones sobre telescopios espaciales, en 1970 estos programas se unieron y en 1971 se creó finalmente "The Large Space Telescope Science Steering Group" (Grupo Directivo de Ciencias del Gran Telescopio Espacial), grupo que se encargaría de llevar a cabo el proyecto. El próximo paso sería conseguir ayuda financiera, la cual debía de ser aprobada por el Congreso de EE.UU., que dijo que no. Por suerte, se invitó a la Agencia Espacial Europea (ESA, en inglés) a formar parte del programa y así lograron reducir los costos a la mitad. De esta manera, el Congreso sí aceptó brindar los fondos para la financiación, y en 1977 el proyecto del telescopio espacial Hubble fue finalmente aprobado.

Fue un trabajo en equipo: la ESA se encargó principalmente de los paneles solares que le darían vida al telescopio mientras éste estuviera en órbita, la NASA se encargó del diseño, desarrollo y construcción del telescopio en sí, y se contrató a la empresa PerkinElmer para la construcción del espejo primario (el ojo del gigante, la principal fuente de detección de luz y un elemento muy importante más adelante en esta historia) y a Lockheed Missiles para fabricar la nave espacial y sus sistemas de apoyo.

El lanzamiento estaba originalmente planeado para 1983 pero (a la obra siempre hay que calcularle un poquito más) algunas demoras en su construcción y ensamblaje, junto al accidente del transbordador espacial Challenger en 1986, donde fallecieron los 7 miembros de la tripulación, ocasionaron que se frenara toda la actividad espacial hasta 1988.

Finalmente, el Hubble fue lanzado a bordo del transbordador espacial Discovery, hace ya 30 años, el 24 de abril de 1990.

\section{Cómo (no) funciona}

El telescopio Hubble es, en esencia, un tubo con dos espejos: un espejo primario (de 2,4 metros de diámetro) que se encuentra en un extremo del tubo y se encarga de recibir todas las ondas de luz, y que las refleja en un espejo secundario, que a su vez las redirige hasta converger en un solo punto y registrar la imagen. Por eso entra en la categoría de los telescopios reflectores.

Pero es mucho más que un tubo con dos espejos. Los instrumentos que lo componen le permiten no sólo detectar la luz del espectro visible (la luz que nuestros ojos pueden ver), sino que también admite longitudes de onda más grandes como las de infrarrojo, o más 
chicas como las de ultravioleta y (ondas que, exceptuando algunas infrarrojas, la atmósfera no deja pasar).

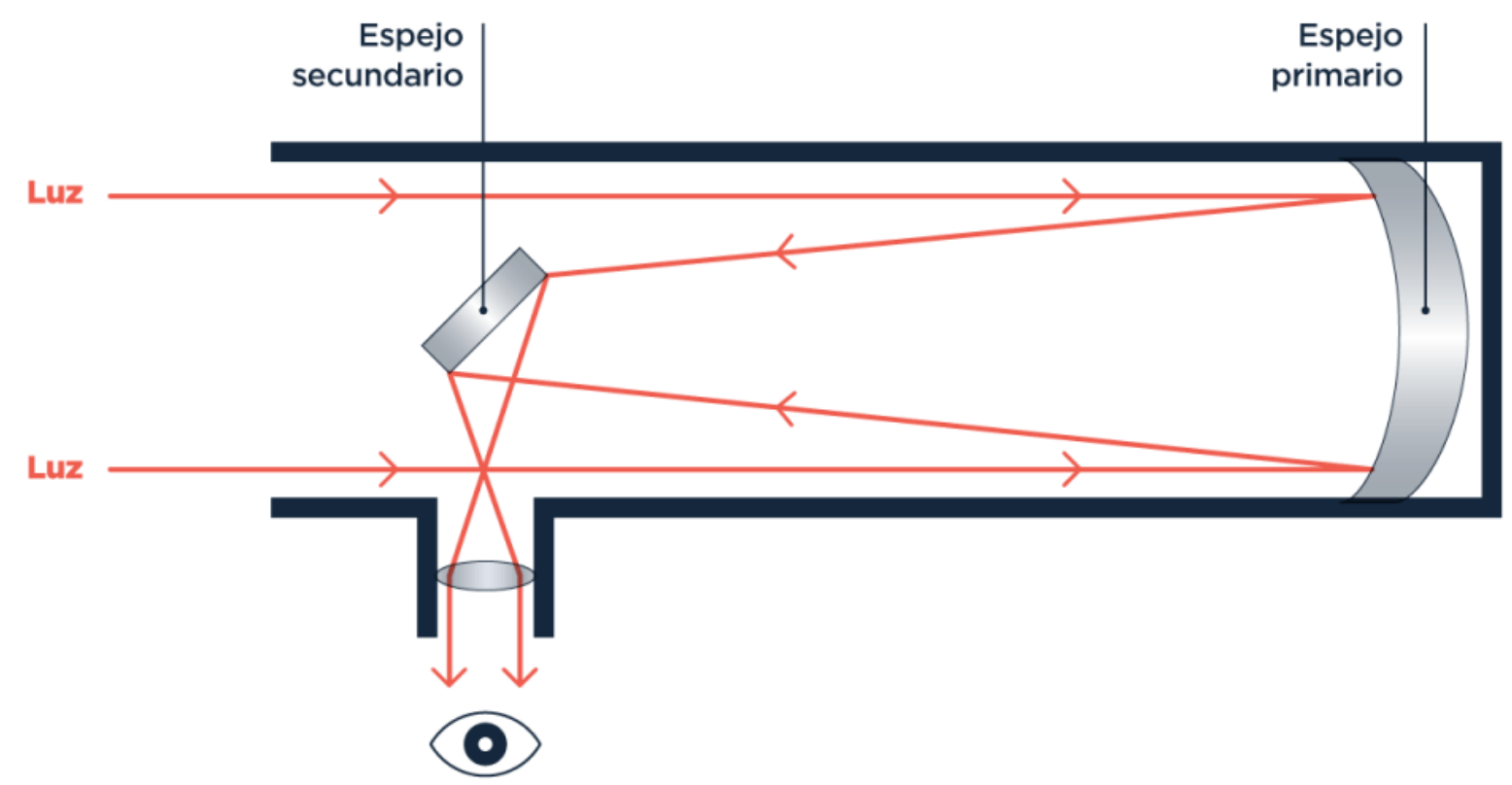

Diagrama (muy) simplificado de cómo funciona un telescopio reflector.

Luego de gastar cientos de millones de dólares en su construcción y ponerlo a orbitar a unos $500 \mathrm{~km}$ de la Tierra, a una velocidad aproximada de $7 \mathrm{~km}$ por segundo, lo ideal hubiese sido que todo funcionara correctamente. Pero pocas semanas después de su lanzamiento, especialistas empezaron a notar que las imágenes obtenidas eran más borrosas (desenfocadas) de lo esperado, y si bien la distorsión aún permitía estudiar en alguna medida al cosmos, dificultaba mucho el trabajo e incluso imposibilitaba sacar provecho de varios de los componentes del aparato. En un primer momento pensaron que el problema se debía a que los giroscopios que se encargaban de mantener al Hubble 'estable', que era uno de los mayores problemas que tenían que resolver previo al lanzamiento, estaban fallando; o sea, si a uno mismo a veces le cuesta sacar una foto y que no salga borrosa, imaginemos esa tarea dando vueltas a la Tierra a miles de kilómetros por hora. Pero luego de investigar un poco llegaron a la conclusión de que la falla se encontraba en el espejo primario, que sufría de una aberración esférica (básicamente, cuando la luz entraba y se reflejaba en el espejo, no todos los rayos de luz convergían en el punto esperado). Un error de principiante. El defecto era de apenas 4 milímetros, suficiente como para tirar por la borda el trabajo de cientos de científicos. 
Gracias a que se pensó que en algún momento el Hubble podría llegar a tener alguna falla, el telescopio fue diseñado con una característica que hasta el día de hoy lo hizo único: estando en órbita, puede ser acoplado a los transbordadores espaciales que utilizó la NASA entre 1981 y 2011 para viajar al espacio, y así recibir tareas de mantenimiento. De esta manera, luego de que los 7 astronautas obtuvieran el entrenamiento necesario y aprendieran a utilizar herramientas nunca antes utilizadas en el espacio, a fines de 1993, despegó la misión STS-61 con el propósito de hacerle chapa y pintura a la imperfección en el espejo primario, junto con la instalación de nuevos instrumentos. La reparación tuvo una duración de 11 días y es considerada por muchos como una de las misiones más complejas en la historia de los viajes espaciales. 


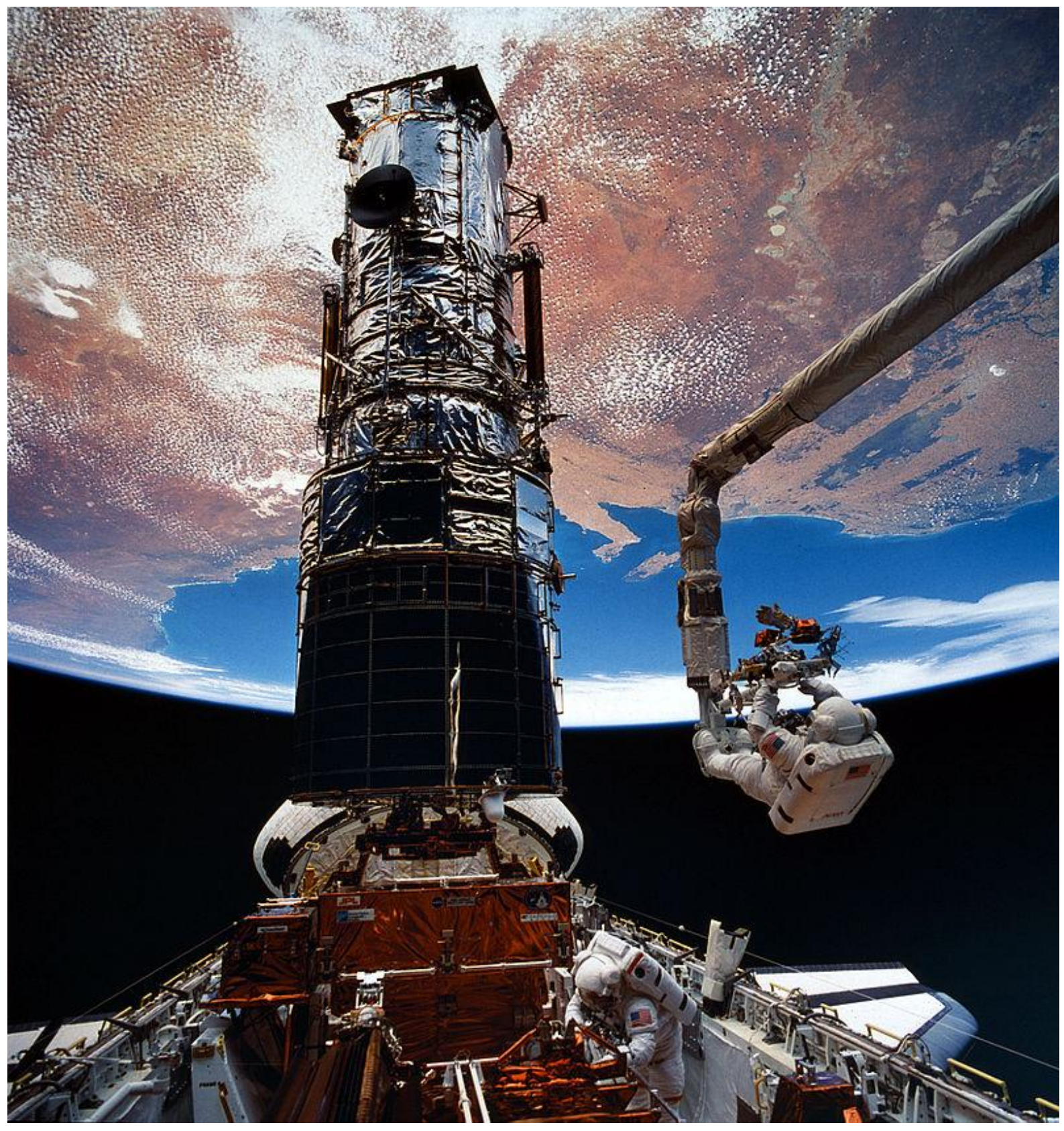

El telescopio Hubble ensamblado al transbordador espacial Endeavour mientras era reparado. Crédito: NASA

Una vez finalizada la misión, se realizaron las calibraciones y ajustes de softwares necesarios para así evaluar el éxito de la reparación. El resultado fue un sí rotundo: 

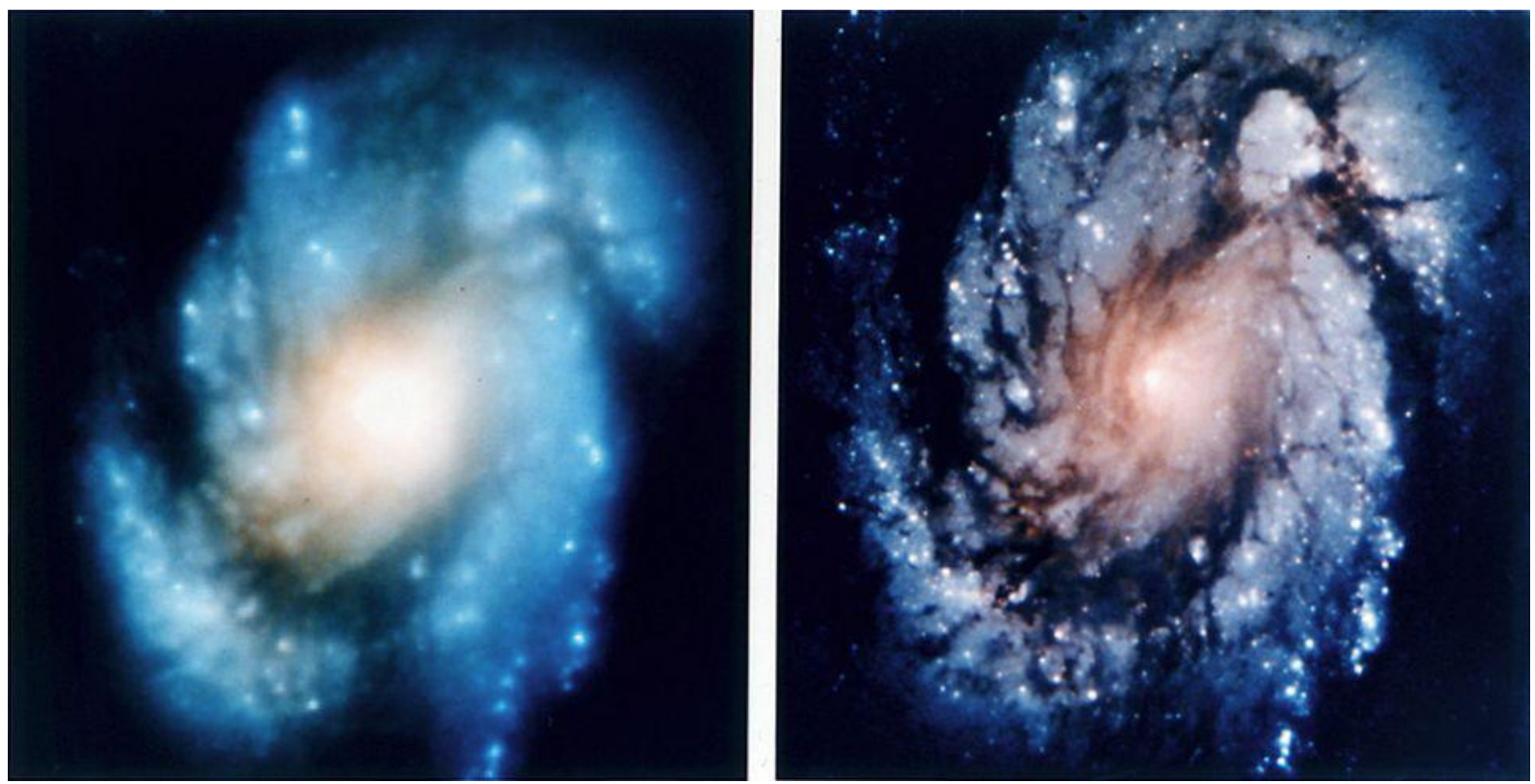

Fotografías de la galaxia M100 tomadas antes y después de la reparación del Hubble. Crédito: NASA

Inmediatamente después de haber sido reparado, astrónomos de todo el mundo (pues para poder utilizarlo 'simplemente' basta con enviar una solicitud a la NASA) empezaron a sacarle el máximo provecho; y no se tardó mucho tiempo en llegar al primer descubrimiento revolucionario, pues a mediados del '94, luego de haber observado la galaxia M87, ubicada aproximadamente a unos 50 millones de años luz, se encontró evidencia acerca de que ésta tenía un agujero negro supermasivo en su centro, algo que ya se sospechaba pero que aún no se había podido demostrar. Este hallazgo proporcionó un apoyo muy fuerte a la existencia de los agujeros negros y ayudó a respaldar la hipótesis de que en todos los centros galácticos existe uno.

Un año después, en diciembre del '95, se publicó el "Hubble Deep Field” (HDF), o Campo Profundo del Hubble, que es una imagen resultante de una serie de observaciones en donde aparecen más de 3000 galaxias, algunas de las cuales se encuentran entre las más viejas y distantes que se conocen. Lo útil de captar galaxias tan lejanas es que nos permite observar cómo era el Universo en sus comienzos ya que, si somos capaces de observar un objeto a, supongamos 13.000 millones de años luz, significa que la luz que estamos captando de él demoró 13.000 millones de años en llegar hasta nosotros. Gracias al HDF se han publicado cerca de 400 artículos científicos. Tres años después, se tomó una imagen de las mismas características pero en una región diferente, la cual fue denominada Campo Profundo Sur del Hubble, y al observar que a gran escala ambas imágenes eran 
muy similares, se reforzó la idea de que el Universo es uniforme si se lo estudia a gran escala (algo de lo que hablamos acá).

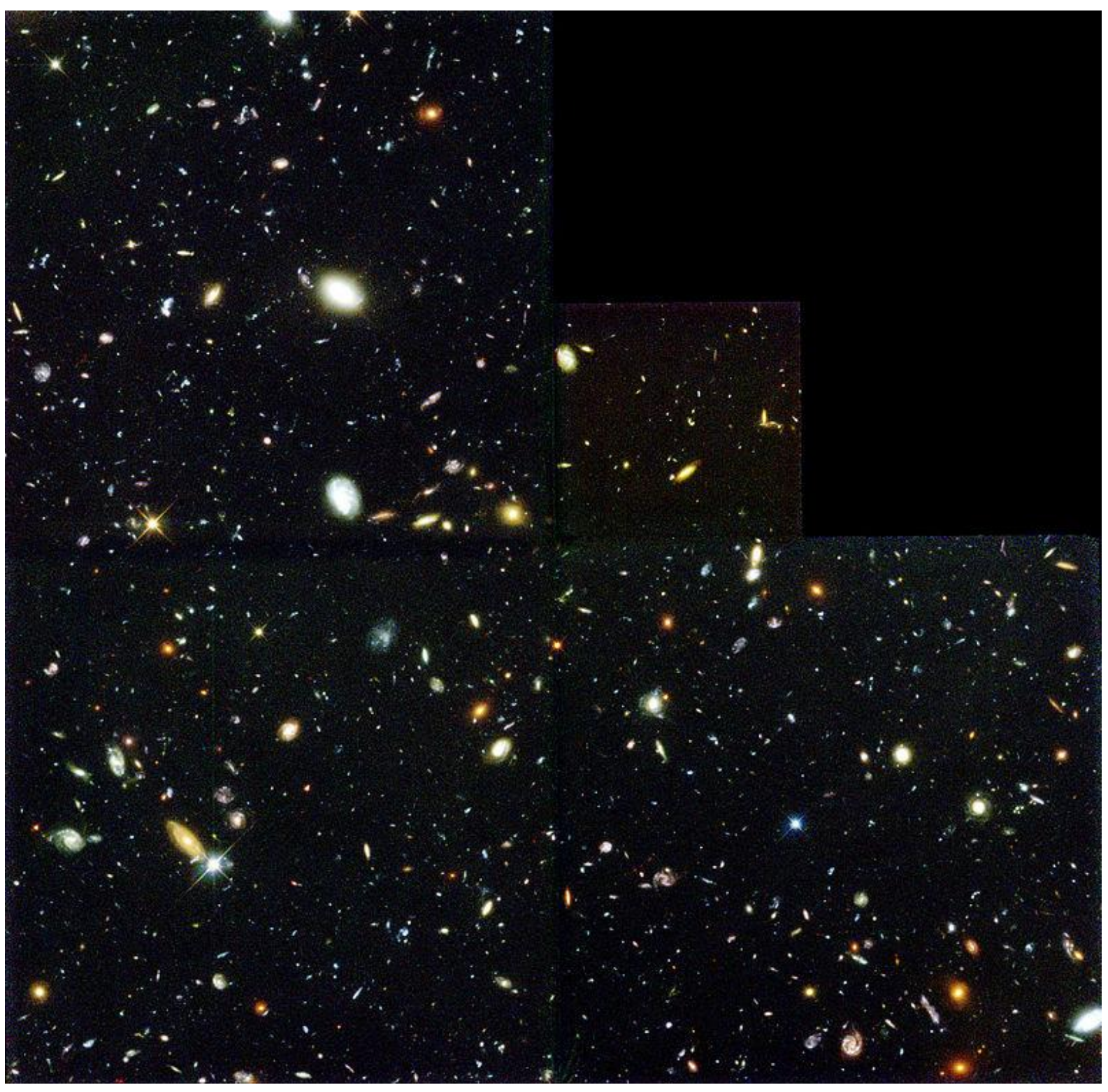

El Hubble Deep Field. Crédito: NASA/ESA

Como dijimos antes, el principal objetivo del Telescopio Hubble era ayudar a determinar la edad del Universo y su velocidad de expansión, dos cosas relacionadas. Partiendo de la idea de que al comienzo todo estaba comprimido en un punto (singularidad), o al menos en una región mucho más pequeña de la que hay hoy en día, para saber la edad del Universo 
sólo habría que calcular cuánto tiempo pasó desde que todo estaba comprimido en esa región, lo cual básicamente depende de cuánto y qué tan rápido se estuvo expandiendo el Universo en todo este tiempo, y esto, a su vez, depende de lo que hoy en día conocemos como Constante de Hubble (que en realidad, no es un número fijo, sino más bien un parámetro que va variando lentamente con el tiempo). La ley de Hubble postula que la velocidad a la cual un objeto se aleja de otro es igual a la distancia en que se encuentran multiplicada por la constante de Hubble; esto indica que cuánto más lejos esté un objeto, más rápido se aleja, lo cual tal vez al principio parece un poco ilógico, pero tiene mucho sentido: si Córdoba se encuentra a 400 km de Rosario y a 700 km de Buenos Aires y expandimos el espacio entre ellas hasta el doble, Rosario pasa a estar a $800 \mathrm{~km}$ (se alejó 400 km) y al mismo tiempo Buenos Aires pasa a estar a 1400 km (se alejó 700 km). Por lo tanto, sólo hay que calcular la constante de Hubble para así poder determinar la tasa de expansión del Universo y con ello su edad. Acá es en dónde nuestro telescopio entra en acción.

¿Cómo se calcula la constante de Hubble? De lo anterior se deduce que esta depende de la velocidad a la que se alejan dos objetos en un instante dado y de la distancia entre ellos en ese instante. Un telescopio espacial es ideal para medir estas dos variables, porque a partir de la luz que recibimos de las estrellas o galaxias que observamos, podemos obtener ambas cosas. La luz de un objeto alejándose de nosotros, se va tornando más 'rojiza', efecto denominado 'corrimiento al rojo' (algo así como el Efecto Doppler, que provoca que cualquier sonido, una sirena por ejemplo, se escuche más grave a medida que se va alejando). Estudiando este fenómeno de la luz, podemos determinar a qué velocidad se está alejando el objeto; además, para calcular la distancia a la cual está ubicado, se compara el brillo que recibimos con ese objeto, con el brillo que recibiríamos si lo tuviéramos justo al frente. Así, gracias al Hubble, se pudo determinar que la expansión del Universo aumenta $70 \mathrm{~km} / \mathrm{s}$ cada 3,6 millones de años luz y que, además, la expansión está acelerando (descubrir esto último les valió el Nobel de física de 2011 a Saul Perlmutter, Adam Riess y Brian Schmidt). Así también se pudo calcular que el Universo tiene aproximadamente $\mathbf{1 3 . 8 0 0}$ millones de años. 


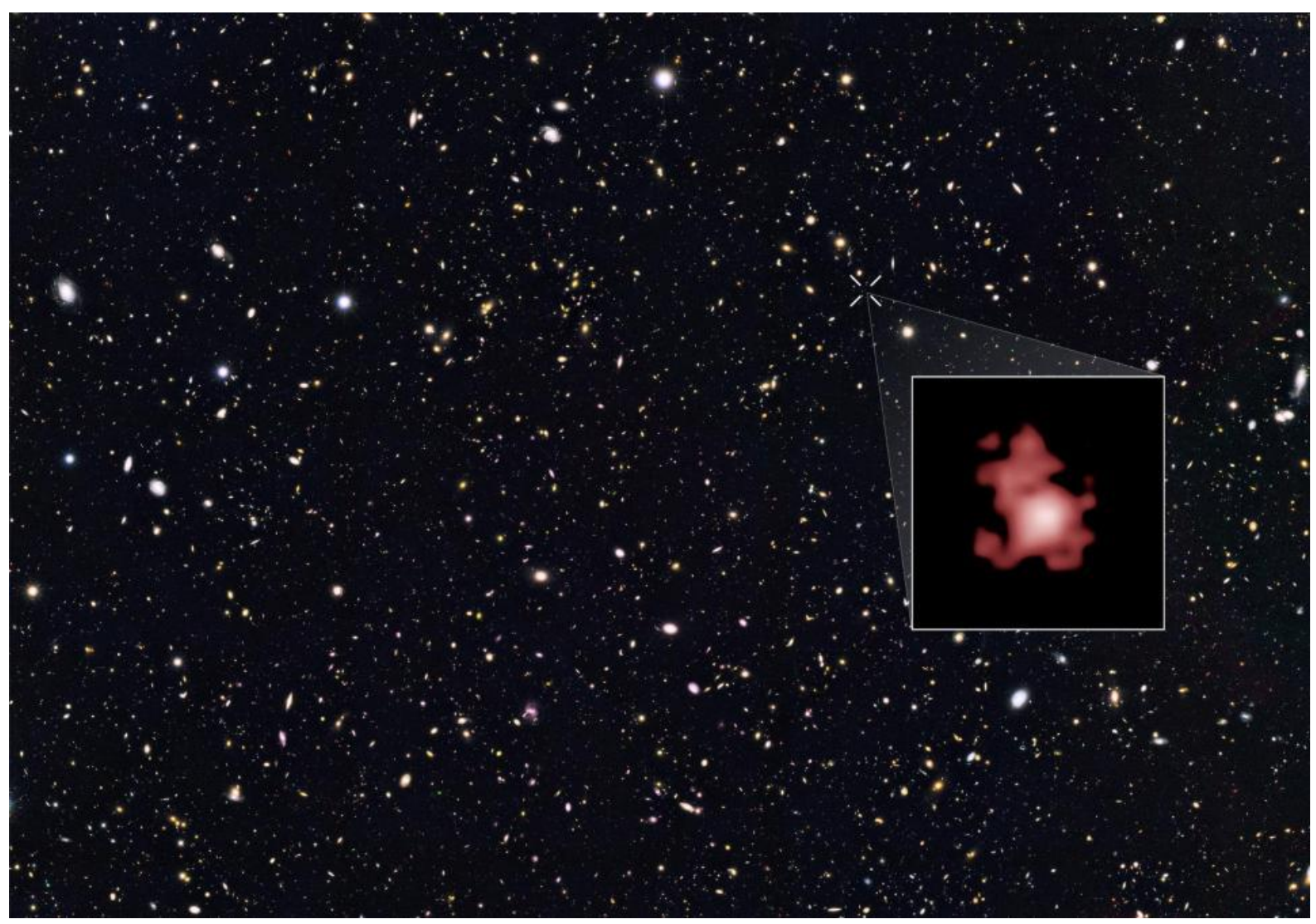

Esa "mancha" roja resaltada en la imagen, es la galaxia GN-z11, fotografiada en 2016 por el Hubble y es hasta ahora la galaxia más distante conocida, ubicada a 13.400 millones de años luz. Al observarla, somos capaces de estudiar cómo era el universo primitivo, con tan solo 400 millones de años de edad. Crédito: NASA/ESA

\section{Las fuerzas ocultas}

Hacia fines de los '90, el hecho de haber detectado que la expansión del Universo estaba en aceleración causó revuelo en la comunidad científica, pues hasta ese momento se teorizaba que la fuerza de la gravedad provocaría que en algún momento esa expansión se detuviera, y lo 'correcto' habría sido detectar que la expansión estaba desacelerando. Esto (junto a otros indicios), le dio vida a una hipótesis que ya había sido mencionada por muchos científicos en décadas anteriores, pero a la cual no se le había prestado mucha atención por la poca evidencia existente hasta el momento: el hecho de que tal vez hubiera un tipo 'raro' de energía o materia que no habíamos sido capaces de detectar hasta el momento y que influyeran en el estado de expansión. Estas 'fuerzas ocultas' son denominadas energía oscura y materia oscura, y hoy se sabe que componen el 68 y $27 \%$ 
del Universo, respectivamente. ¿Esto quiere decir que toooodos los objetos que fuimos capaces de observar a lo largo de nuestra historia, como estrellas, planetas, galaxias enteras, etc., apenas componen el $5 \%$ del Universo? Sí. Y como no podía ser de otra manera, el Hubble brindó su granito de arena a este misterio que aún hoy continúa, pues ayudó a diseñar un mapa con la distribución a gran escala de la materia oscura, estudiando la influencia gravitatoria de ésta en objetos que sí podemos observar.

También se usó para mejorar nuestro entendimiento acerca de las zonas de formación de nuevos planetas, que son lo que se denominan 'discos protoplanetarios' y se encuentran alrededor de las estrellas. Otro beneficio: creció nuestro entendimiento acerca de las estaciones y cambios climáticos en otros planetas que no sean la Tierra.
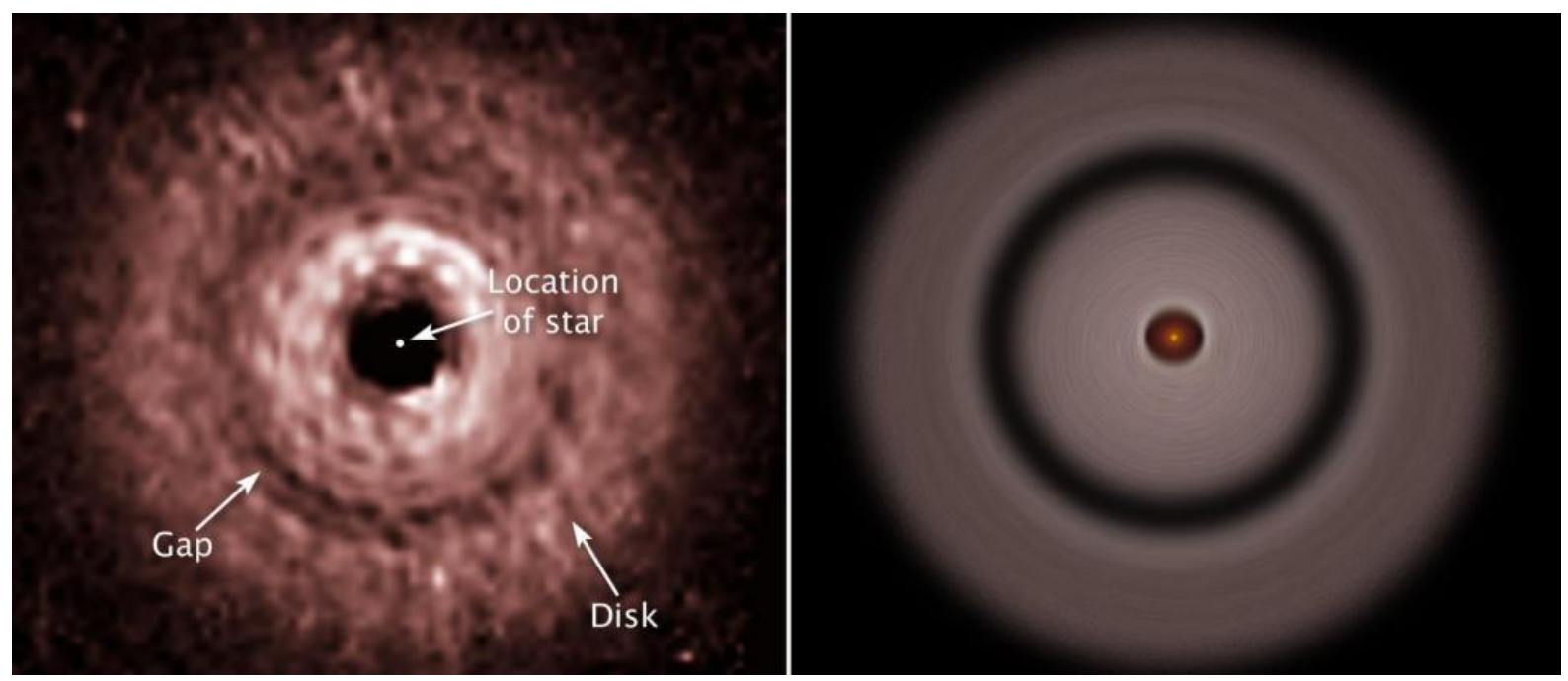

Disco protoplanetario captado por el telescopio Hubble de la estrella TW Hydrae, ubicada a tan solo 196 años luz. Crédito: ESA/NASA

Si tuviéramos que seguir nombrando todos los descubrimientos hechos con el Hubble, esta nota se haría interminable ya que gracias a él se han publicado más de 15.000 artículos científicos que hablan sobre alguna porción del universo. El telescopio Hubble ha ocasionado una enorme revolución en la astronomía, muchas veces reafirmando en observaciones lo que se había previsto con la teoría, pero también en incontables ocasiones dejó boquiabiertos a muchos debido a que los resultados no eran los esperados. Hasta el día de hoy, 30 años después de su lanzamiento, sigue ayudándonos a descubrir los secretos mejor guardados de nuestro Universo. 
En toda su historia, el Hubble recibió 5 servicios de mantenimiento, el último en 2009, y se espera que siga operando hasta mediados del 2030. Por semana, transmite más de 120 gigabytes de datos científicos. $Y$ hay buenas noticias: la NASA tiene planeado lanzar el 30 de marzo del 2021 el telescopio espacial James Webb, el cual va a tener un espejo primario 5 veces más grande que el del Hubble, lo que permitirá hacer mediciones y observaciones aún más precisas.

El Hubble nos ha dado la edad del Universo, nos ha enseñado sobre las distancias, nos ha acercado a los objetos que teníamos lejos y ha echado luz sobre algunas de las zonas más oscuras del Universo. Y, como si eso fuera poco, nos ha regalado algunas de las postales más hermosas de ese misterio infinito que está ahí afuera:

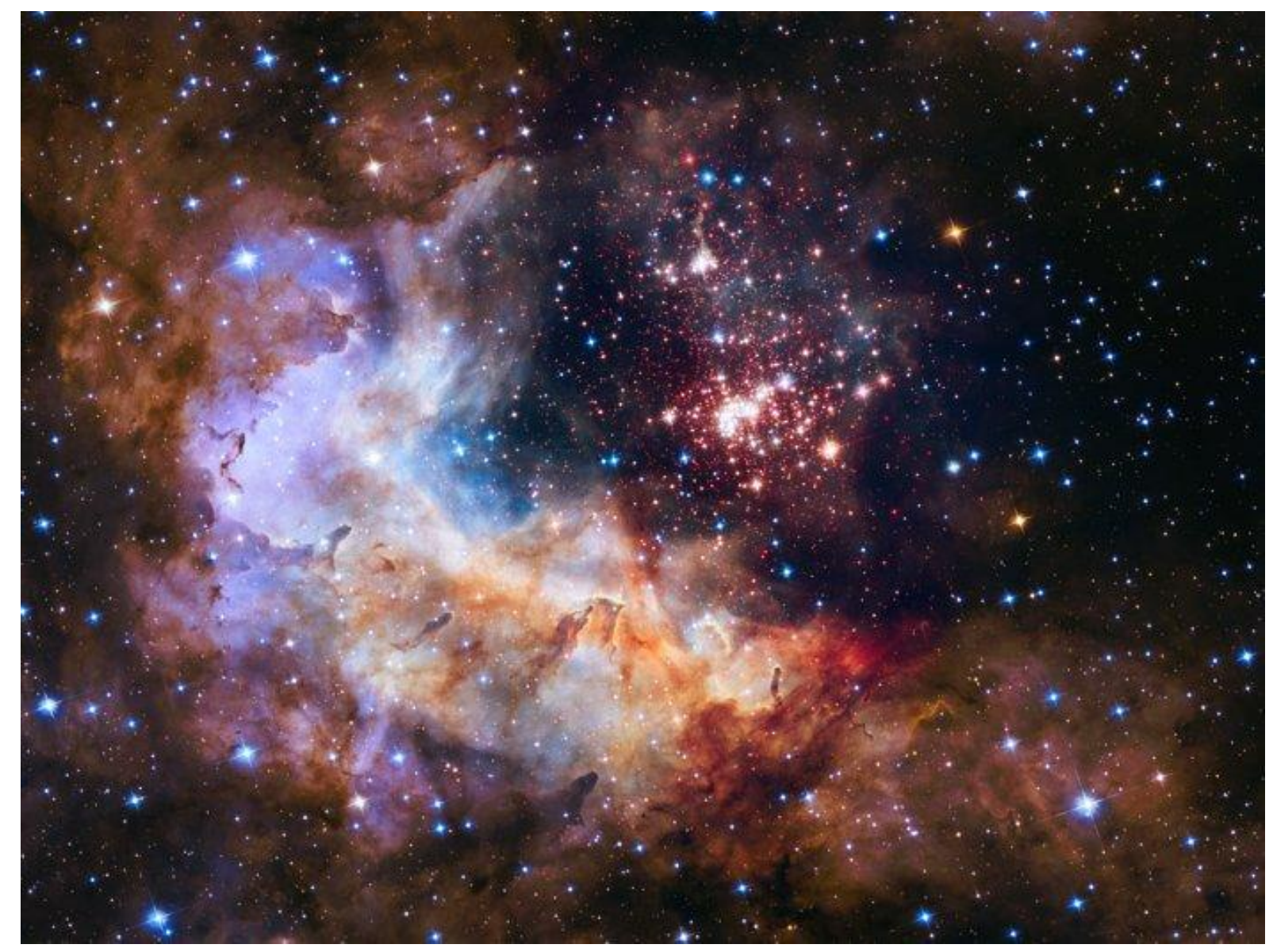

Westerlund 2, un cúmulo de estrellas en la Vía Láctea ubicado a 20.000 años luz de distancia. Contiene las estrellas más masivas y brillantes de la galaxia. Crédito: NASA/ESA. 


\section{JOSHA}

Journal of Science, Humanities and Arts

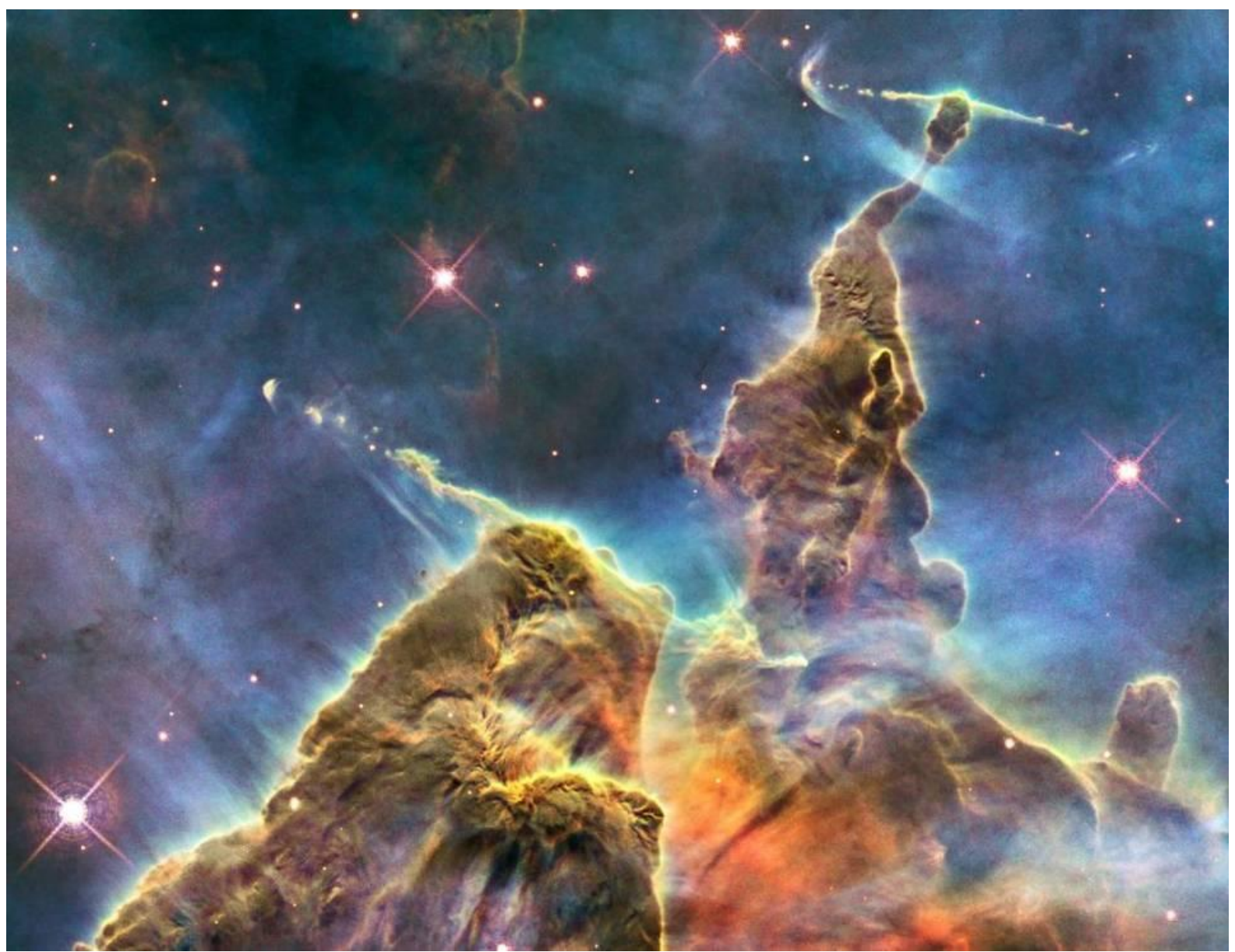

Nebulosa de la Quilla, se encuentra a una distancia aproximada de 8.000 años luz. Crédito: NASA/ESA 


\section{JOSHA}

Journal of Science, Humanities and Arts

August 2021

Volume 8, Issue 4

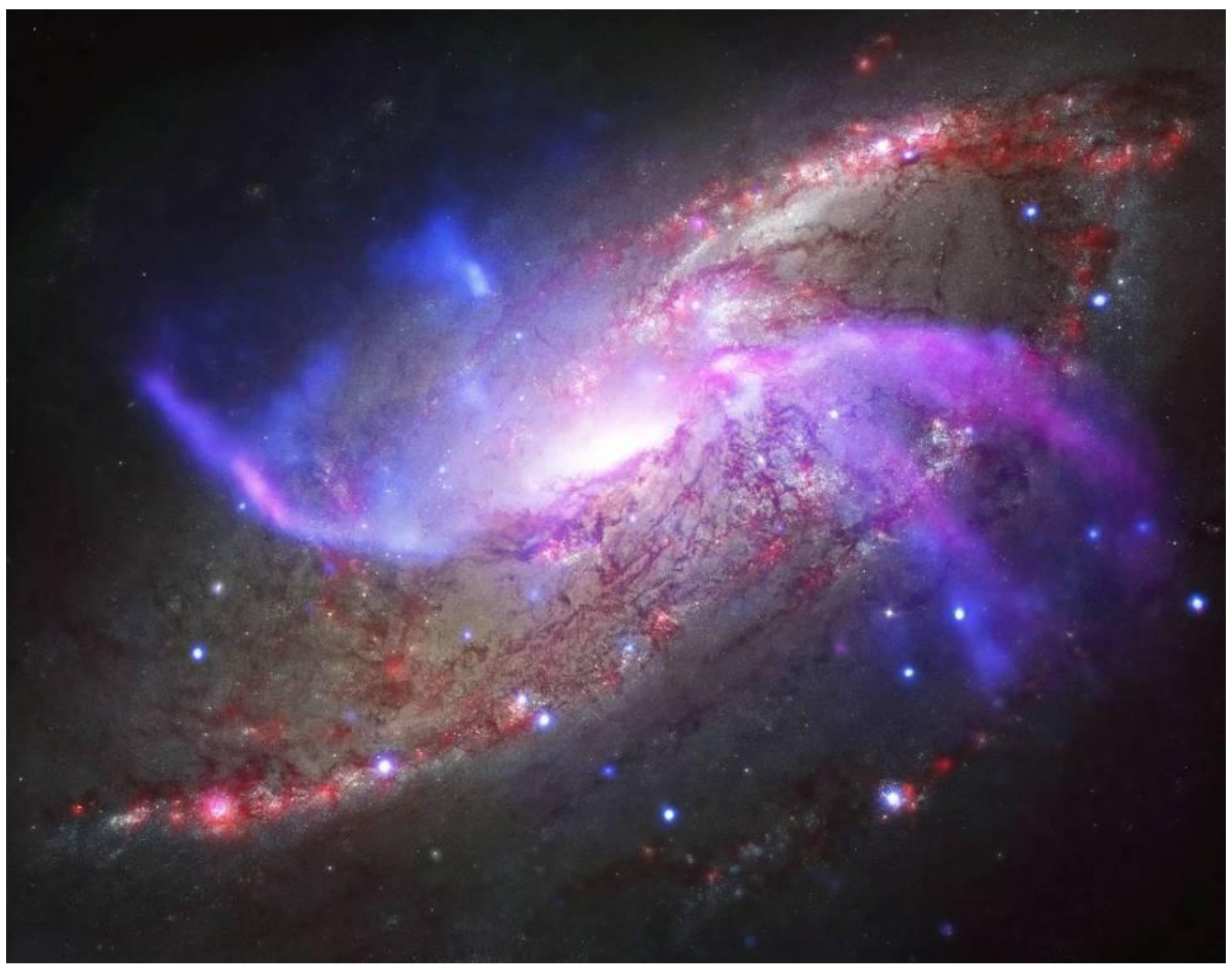

Galaxia M106, ubicada a 22 millones de años luz. Crédito: NASA/Caltech 


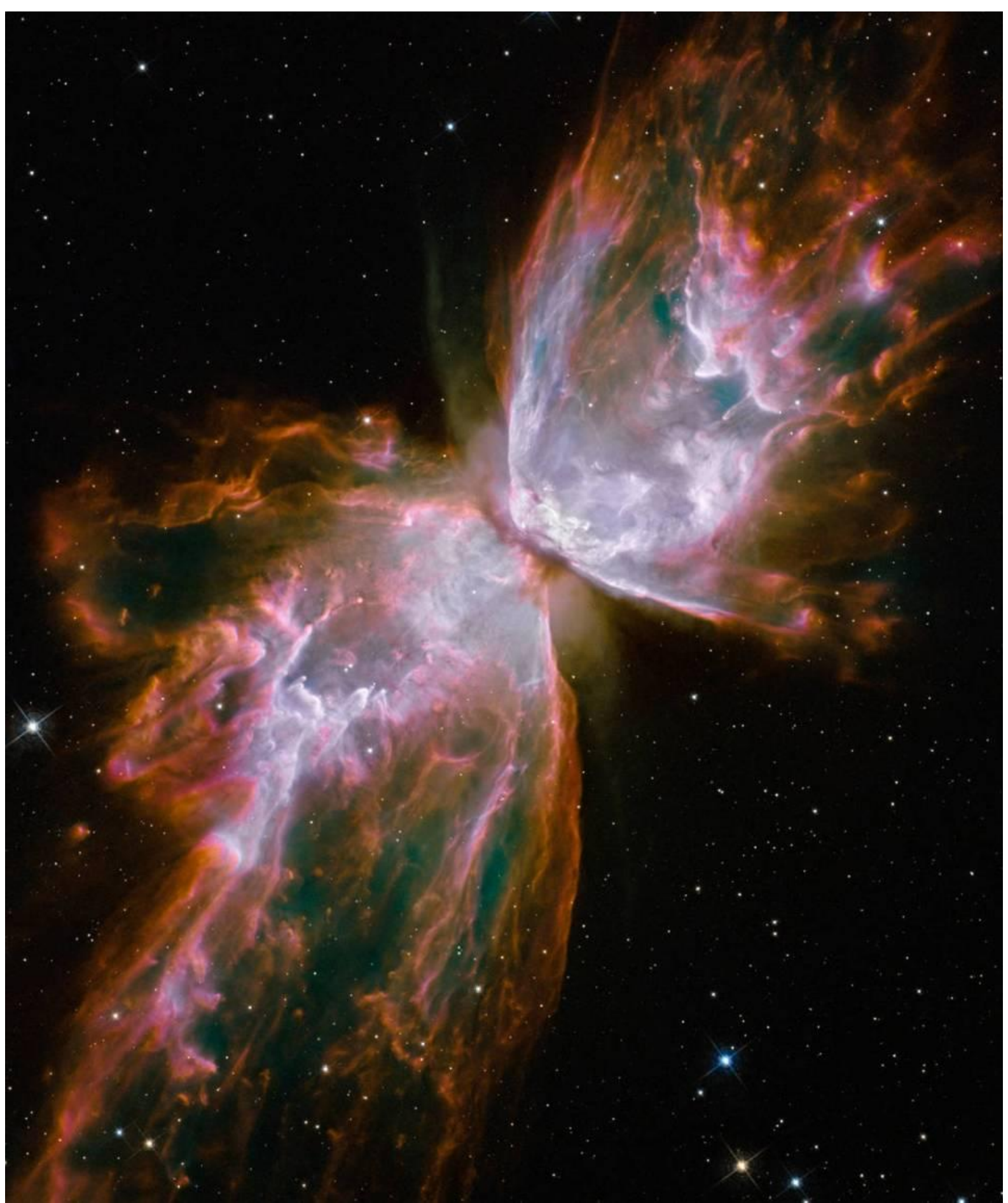

Nebulosa de la mariposa, ubicada a 3.200 años luz de distancia, es de las nebulosas más complejas que se conocen hasta el momento. Su estrella central, es uno de las estrellas más calientes conocidas. Crédito: NASA/ESA 


\section{Referencias}

https://www.nasa.gov/mission_pages/hubble/story/index.html

https://www.nasa.gov/content/goddard/2017/highlights-of-hubble-s-exploration-of-the-univ erse

https://hubblesite.org/

https://web.archive.org/web/20080327091202/http://www.spitzer.caltech.edu/about/spitzer .shtml

https://history.nasa.gov/hubble/

https://www.space.com/3319-astronomers-create-3d-map-dark-matter.html

https://mashable.com/2015/04/24/hubble-telescope-discoveries/

https://www.theguardian.com/science/2016/jun/03/universe-is-expanding-up-to-9-faster-th an-we-thought-say-scientists

https://www.youtube.com/watch?v=-nNiULI5_2k\&list=PLogT2Nw88-2PkSW9e87XyuHiSwC $-\mathrm{wXHg} 1$ \&index $=2 \& \mathrm{t}=6 \mathrm{~s}$

https://www.space.com/17-amazing-hubble-discoveries.html

https://www.nasa.gov/mission_pages/hubble/multimedia/index.html 